\title{
A STUDY OF X-RAY CHEST PATTERNS IN MULTIDRUG-RESISTANT TUBERCULOSIS (MDR-TB) BETWEEN HIV REACTIVE AND HIV NON-REACTIVE PATIENTS IN A TERTIARY HILLY MEDICAL CENTRE
}

\author{
Sujit Kumar Bhattacharyya ${ }^{1}$, Pratik Barma², Rajarshi Bhattacharyya ${ }^{3}$ \\ ${ }^{1}$ Associate Professor, Department of Chest Medicine, Darjeeling North Bengal Medical College, West Bengal, India. \\ ${ }_{2}^{2}$ RMO-Cum-Clinical Tutor, Department of Chest Medicine, Darjeeling North Bengal Medical College, West Bengal, India. \\ 3Junior Resident, Department of Chest Medicine, KPC Medical College, Jadavpur, Kolkata, West Bengal, India.
}

\begin{abstract}
BACKGROUND
Globally, multidrug resistant-tuberculosis (MDR) is one of the major healthcare problems. India is not far away from that concern especially when there is an increasing association with human immunodeficiency virus (HIV). As multidrug resistant tuberculosis (MDR-TB) is determined by microbiological testing, early detection of MDR -TB by X-Ray chest is highly recommended to help in guiding diagnostic confirmation. The chest $\mathrm{x}$-ray patterns in MDR-TB depends on multiple risk factors including immune status and therefore identifying the influences of HIV on chest $x$ ray appearance may help in improving diagnosis.

The objectives of the study were- 1. to describe the chest x-ray patterns in patients with MDR-TB and 2. To compare the radiological pattern in X-Ray chest amongst MDR-TB patients between HIV reactive and HIV non-reactive group.
\end{abstract}

ABSTRACT

\section{MATERIAL AND METHODS}

A descriptive cross-sectional study was conducted among 80 patients in the chest department of a rural tertiary care hospital in northern West Bengal during the period of 1st Dec 2016 to 30th Nov 2018. All patients were diagnosed as MDR-TB by cartridge based nucleic acid amplification test (CBNAAT) or GeneXpert. In all patients, digital x-ray chest PA and lateral view were done routinely. Side by side HIV status was assessed in all patients. Statistical analysis was performed using IBM SPSS statistics software.

\section{RESULTS}

Among 80 patients who were included in our study, male to female ratio was 5:3. Majority belonged to labour group 32.5\%. Major radiological abnormalities found were cavitary lesions (44.12\%) followed by consolidation (32.35\%) in HIV non-reactive group. Whereas in HIV reactive group hilar lymphadenopathy (50\%) was predominant and cavitary lesion and consolidation were less common.

\section{CONCLUSION}

The most common chest x-ray finding was cavitary lesion followed by consolidation especially in HIV non-reactive group. In HIV reactive group, hilar lymphadenopathy was predominant finding. Though cavitation in adult may raise the possibility of tuberculosis, it does not exclude MDR tuberculosis especially if it is multiple in number.

\section{KEY WORDS}

Chest X-Ray, Multi Drug Resistant Tuberculosis, HIV/AIDS.

HOW TO CITE THIS ARTICLE: Bhattacharyya SK, Barma P, Bhattacharyya R. A study of x-ray chest patterns in multidrug- resistant tuberculosis (MDR-Tb) between HIV reactive and HIV non-reactive patients in a tertiary hilly medical centre. J. Evolution Med. Dent. Sci. 2019;8(06):389-393, DOI: 10.14260/jemds/2019/86

\section{BACKGROUND}

In March 2017, the Government of India (GOI) announced the elimination of Tuberculosis by 2025. India is the country with highest burden of Tuberculosis and Multidrug-Resistant TB (MDR-TB). There are an estimated 79000 MDR-TB patients amongst notified cases of pulmonary tuberculosis each year. India is also second country after South Africa with HIV associated tuberculosis. In 2016 an estimated 28 lakhs cases were found and 4.5 lakhs people died due to tuberculosis.

'Financial or Other Competing Interest': None.

Submission 13-01-2019, Peer Review 02-02-2019,

Acceptance 40-02-2019, Published 11-02-2019.

Corresponding Author:

Pratik Barma,

C/o. Dr. Sujit Kumar Bhattacharyya,

Vill Aminpur, PO Khamarchandi,

PS: Haripal District Hooghly-712405,

West Bengal,

India.

E-mail: drsujit.haripal@yahoo.in

DOI: $10.14260 /$ jemds $/ 2019 / 86$ 2016 out of Which 56 percent of tuberculosis found in 5 countries like India, Indonesia, China, Philippines and Pakistan. ${ }^{1} \mathrm{HIV}$ infection is a potent risk factor for tuberculosis either by activation of latent tuberculosis or by undermining the cell mediated immunity (CMI) through depletion of CD4 lymphocytes. ${ }^{2}$ Immunosuppression results in lack of ability of the body to fight against infections leading to increased susceptibility to various opportunistic agents. Lung is one of vulnerable organ. India is home to approximately 2.5 million people living with HIV, the largest number of cases of any country in the world and is recognised as the source of increasing HIV prevalence among its south Asian neighbours. Recently released national HIV prevalence estimates for India indicate that $0.22 \%$ of woman and $0.36 \%$ of men aged $15-49$ years are infected. ${ }^{3}$

During anti tubercular drug (ATD) therapy there is a selection pressure on the population of Mycobacterial tuberculosis ( $\mathrm{M} \mathrm{Tb}$ ) results in the occurrence of spontaneous resistance causing mutations in the susceptible bacilli which then gradually increases to become dominant strain. ${ }^{4}$ 
Initially frequency of single mutation is very low and that may increase gradually to become dominant strain. This problem can be overcome if appropriate combination of chemo therapy is administered either in drug sensitive pulmonary tuberculosis or in drug resistant tuberculosis (DR TB).

Multi-drug resistant tuberculosis (MDR-TB) refers to tubercular infection resistant to rifampicin and isoniazid with or without other first line of anti-tubercular drugs.

In 2016, there were 600000 new cases with resistance to rifampicin, the most effective first line anti tubercular drug of which 490000 had MDR-TB and almost $47 \%$ cases were found in India, china and the Russian federation. There were 476774 reported cases of HIV reactive cases with tuberculosis. Irregular and inappropriate use of medication and HIV/TB co infection contributes increased concern when resistant mutant arises during treatment with first line antitubercular drugs (ATD), it is considered as acquired resistant tuberculosis. People who are infected by already drug resistant strain called primary resistant tuberculosis who are seen amongst those people not taking anti tubercular drug at all.

It has been seen that globally $3.5 \%$ of newly diagnosed TB patients and $20.5 \%$ of previously treated patients had MDR TB. 5 Treatment success rate of patients with drug resistant MDR TB is only $48 \%$. Mortality rate ranged from $1 \%$ to $30 \% .6$

Prevention and early diagnosis of MDR-TB are priorities for proper MDR-TB control. Previously for diagnosis of drug resistant tuberculosis several weeks were required but now with the introduction of newer investigation and diagnostic approach like cartridge based nucleic acid amplification test (CBNAAT) has decreased the delay in diagnosis but these testing facilities are not available at every corner of our country and non- availability of cartridge sometimes further delay in the diagnosis of MDR TB. Thus, suspicion of diagnosis of drug resistant tuberculosis by chest imaging can further guide and even intensify diagnostic approach for MDR TB.

\section{MATERIALS AND METHODS}

The present study is a descriptive study with cross sectional design. All patients (Complete enumeration) with MDR TB who attended the indoor and out Patient Departments (OPD) of chest medicine of a rural tertiary care hospital in north of West Bengal, India, between $1^{\text {st }}$ December 2016 to 30th November 2018 were consecutively enrolled in the study. Multi-drug resistant tuberculosis (MDR-TB) as defined by resistance to both isoniazid and rifampicin and or other first line of anti-tubercular drugs (ATD). They were diagnosed from samples of sputum, lymph node, CSF, discharge from sinus tract as and when required using following methods:

1. In most of the cases MDR TB was diagnosed on the basis of Cartridge based nucleic acid amplification test (CBNAAT).

2. Molecular testing using GeneXpert/PCR.

Data were collected through chart review under name, age, sex, occupation, drug resistant study as evidenced from CBNAAT and or PCR/Gene expert study, digital x-ray PA and lateral view, HIV status. Ultrasonography of chest was done in selected cases to differentiate between pleural effusion and pleural thickening.
The chest X-ray-was reviewed by the same senior consultant radiologist who read chest $\mathrm{x}$ rays systematically with a standardized recording. The reporting radiologist was blinded to the HIV status.

Chest x-ray was systematically reviewed for the presence of following variables

1. Hilar/mediastinal lymphadenopathy described as nodular masses according to the sites.

2. Segmental/lobar consolidation described as a homogenous increase in pulmonary parenchymal attenuation that obscures the margin of vessels and air way walls.

3. Cavities described as gas filled spaces seen as a lucency or low attenuations are with pulmonary consolidation.

4. Miliary opacities described as a profuse tiny, discrete rounded pulmonary opacities $\leq 3 \mathrm{~mm}$ in diameter that are uniformly distributed throughout the lung

5. Fibrosis described as ground glass opacities, fine nodular shadows, linear shadows and reticular shadows (Small or Large)

Statistical analysis was performed using IBM SPSS statistics software. For continuous variables such as age, the mean, median and range were calculated. Results were recorded as frequencies and percentages for categorical variables. The Pearson chi square test or fisher exact test (where the expected frequencies were less than 5) were used to test for significant relationships between the frequency of each $\mathrm{x}$ ray chest patterns and the age. The level of significance was set at $\mathrm{p}<0.05$. The odd ratio(R) and $95 \%$ confidence Interval (CI) were estimated using univariate logistic regression of SPSS.

\section{RESULTS}

80 patients of MDR-TB were selected in our study after confirmation by CBNAAT/GENE X-PERT. All patients who were included in our study were from either indoor or outpatient Departments (OPD) of pulmonary medicine of North Bengal Medical College in West Bengal, India during the period of 1st Dec 2016 to 30th Nov 2018. Out of 80 patients, male was predominant than female with the ratio of 5:3(table 1). Most 46 patients (57.5\%) patients belonged to the age category of $21-40$ years followed by $41-60$ years age group 20 patients $(25 \%)$, less or equal to 20 years age group in $12(15 \%)$ and last group above 61 yrs. age group in 2 (2.5\%) (table 2). Age varied from 10 yrs. to 80 years. Overall average age of patients was 35.45 yrs. Occupationally most 26 patients (32.5\%) patients belonged to labour group found in preferably they came from local tea gardens. Cultivators and drivers contributed $2(2.5 \%)$ and $10(12.5 \%)$ patients respectively. House wife found in 14 (17.5\%), students $12(15 \%)$, businessman in 6 cases $(7.5 \%)$ service $10(12.5 \%)$ of which in last group majority belonged to health fraternities (Table 3). 68 patients (85\%) were serologically HIV nonreactive and 12 patients were HIV reactive (15\%) (Table 4) Amongst HIV reactive patient's age varied from 27 to 55 years and average age was $35.67 \mathrm{yrs}$. and majority were male with the ratio 5:1. Most of them were labourers by occupation. All females under this category were victim and got HIV infection from their sexual partner (Table 4). 
Radiologically we categorized all x-ray chest findings into cavitary lesion, consolidation, hilar lymphadenopathy, miliary shadow, fibrotic scar and normal group and these findings were compared separately into two broad headings under HIV non-reactive and HIV reactive groups.

In HIV non-reactive group, predominant radiological findings were cavitary lesion found in 30 cases (44.12\%) either as a isolated lesion or in combination with other radiological abnormalities like pleural thickening in 4 cases and hydropneumothorax in 4 cases. Single cavities found in 10 cases $(33.33 \%)$. Amongst solitary lesion most of the cavity found in Left upper zone followed by left lower zone and right upper zone respectively with the ratio 3:1:1. Multiple cavities were found in 20 cases $(66.67 \%)$ of which bilateral involvement was found in 16 cases $(80 \%)$ and same sided lesions were found in 4 cases (20\%). Bilateral cavities were more predominantly distributed in both upper zone and marginally more on right upper zone $(40 \%)$ as compared to left upper zone (26.67\%) and left lower zone (26.67\%). In HIV reactive group however cavitary lesions were found only in 2 cases $(16.67 \%)$ and all were present on left side with equally distributed in upper and lower zone. Second most common radiological abnormalities that were found in HIV non-reactive group was consolidation among 22 cases $(32.35 \%)$ as an isolated lesion or in combination with other radiological abnormalities like pleural thickening found in 2 cases $(9.09 \%)$, pleural effusion in 2 cases $(9.09 \%)$, hydropneumothorax found in 2 cases (9.09\%). Consolidation predominantly found in upper zone marginally more on right side as compared to left with the ratio 8:5. Bilateral involvement seen in 6 cases $(27.27 \%)$. Comparing with HIV reactive group where consolidation was found in 2 cases $(16.67 \%)$ and located on right side with equal distribution in upper and mid zone. Miliary mottling found in 4 cases (5.88\%) in HIV non-reactive group whereas in HIV reactive group it was $2(16.67 \%)$. Hilar lymphadenopathy exclusively found in 6 cases (50\%) in HIV reactive group. Out of which unilateral lymphadenopathy found in 4 cases of which 3 were in right side and 1 in left side and 2 cases it was bilateral. Normal x-ray found in 2 cases (2.94\%) in HIV non-reactive group. Pulmonary fibrosis was seen in 10 cases $(14.71 \%)$ in HIV non-reactive group. All are bilateral and majority were found in both upper zones. None was found in HIV reactive group (Table 5).

\begin{tabular}{|c|c|}
\hline Sex & Number (\%) \\
\hline Male & $25(62.5 \%)$ \\
\hline Female & $15(37.5 \%)$ \\
\hline \multicolumn{2}{|c|}{ Table 1. Sex $\boldsymbol{n = 8 0}$} \\
\hline
\end{tabular}

\begin{tabular}{|c|c|}
\hline Age & Number (\%) \\
\hline$\leq 20$ Years & $12(15 \%)$ \\
\hline $21-40$ & $46(57.5 \%)$ \\
\hline $41-60$ & $20(25 \%)$ \\
\hline$\geq 60$ & $2(2.5 \%)$ \\
\hline \multicolumn{2}{|c|}{ Table 2. Age Distribution $\mathbf{n}=\mathbf{8 0}$} \\
\hline
\end{tabular}

\begin{tabular}{|c|c|}
\hline House Wife & $14(17.5 \%)$ \\
\hline Student & $12(15 \%)$ \\
\hline Cultivator & $2(2.5 \%)$ \\
\hline Driver & $10(12.5 \%)$ \\
\hline Labour & $26(32.5 \%)$ \\
\hline Businessman & $6(7.5 \%)$ \\
\hline Service & $10(12.5 \%)$ \\
\hline \multicolumn{2}{|c|}{ Table 3. Occupation $\mathbf{n = 8 0}$} \\
\hline
\end{tabular}

\begin{tabular}{|c|cc|}
\hline HIV Reactive & $12(15 \%)$ \\
\hline HIV Non- Reactive & $68(85 \%)$ \\
\hline \multicolumn{2}{|c|}{ Table 4. Immune Status n=80 } \\
\hline
\end{tabular}

\begin{tabular}{|c|c|c|c|c|}
\hline Radiological Presentation & HIV Reactive & HIV Non-Reactive & z Value & p Value \\
\hline Consolidation & $2(16.67 \%)$ & $22(32.35 \%)$ & 0.75 & 0.45 \\
\hline Cavitary Lesion & $2(16.67 \%)$ & $30(44.12 \%)$ & 1.47 & 0.14 \\
\hline Hilar Shadow & $6(50 \%)$ & 0 & 5.47 & $0.00^{*}$ \\
\hline Normal & 0 & $2(2.94 \%)$ & -0.40 & 0.69 \\
\hline Miliary & $2(16.67 \%)$ & $4(5.88 \%)$ & 0.71 & 0.48 \\
\hline Lung Fibrosis & 0 & $10(14.71 \%$ & 0.95 & 0.34 \\
\hline Total & $\mathbf{1 2}$ & $\mathbf{6 8}$ & \\
\hline \multicolumn{7}{|r|}{ Table 5. Radiological Presentation $\mathbf{n}=\mathbf{8 0}$} \\
\hline
\end{tabular}

\section{DISCUSSION}

80 patients were included in our study who were diagnosed to have MDR TB based on cartridge based nucleic acid amplification test CBNAAT/GeneXpert. In our study, majority of patients were males. Aziza et al found male preponderance of MDR TB in their findings. ${ }^{7}$ In our findings age varied from 10 years to 80 years with mean age 35.45 years which was close to Aziza et al with 39.87 years. Deesuwan $\mathrm{P}$ et al described near close observation to their study. ${ }^{8}$ jihoon cha and his associates in their study found mean age amongst MDR TB patients tobe 38 years and age ranges from 15-74 years. ${ }^{9}$

In our observation, most predominant radiological abnormality in HIV non-reactive group was cavitary lesion found in 30 cases (44.12\%). Majority were multiple in 
number (66.67\%). Bilateral cavities were more predominant in both upper zones. Chuchottaworn observed that characteristics of cavities including number of cavities $\geq 3$, the presence of cavities $\geq 2$ lung zones were associated with lung multi-drug resistant- tuberculosis (MDR TB) ${ }^{10}$. Manikkam S et al found cavitary lesion in $31.1 \%{ }^{11}$. Dholakia yn and his associates found an association between cavitation and MDR $\mathrm{TB}$ and they opine bilateral cavities were more common in MDR TB patients ${ }^{12}$. Similar observation found in other study ${ }^{9}$ In contrast in our study in HIV reactive group cavitary lesions were found less as compare to HIV non-reactive group. Jihoon cha and associates found in their study that cavity was less frequent in HIV patients. Long et al,13 Pitchenik observed observation of less cavity in HIV reactive patients. ${ }^{14}$ Kiyan E et al opine about cavity is slightly less in immunecompromised patients than non-immune-compromised group. ${ }^{15}$ Nunes did not found any cavity in 10 MDR TB patients who were HIV reactive patients. ${ }^{16}$ Deesuwan also observed more cavity in their study. ${ }^{8}$

In our study second-most common radiological presentation was consolidation found in $32.35 \%$ cases in non-reactive group. It was predominant in right side as compared to left side and mostly situated in upper zone. Manikkam $\mathrm{S}$ et al found consolidation in about $53.5 \%$ patients. ${ }^{11}$ Aziza found consolidation in $57.4 \%$ and most of the lesion was predominant in right upper zone similar to our study. Contrary Deesuwan and his associates found less consolidation. $^{8}$ In contrast in HIV reactive group consolidation was found less in $16.67 \%$ cases. Paucity may be due to immunosuppression. Kiyan observed $20.6 \%$ lobar or segmental consolidation in his immune compromised group. However, lung fibrosis found in our study in 10 cases $(14.70 \%)$ all were predominant in both sides of lungs. Majority again found in upper zone. Deesuwan and his associates found fibrosis to be more common form of radiological presentation in MDR TB in non-reactive HIV group. Aziza et al found fibrosis in lung in $23 \%$ cases and situated predominantly found in upper zone preferably in right side. Though we could not find any side predilection in our study.

Hilar lymphadenopathy was found in 6 cases (50\%). All belonged to HIV reactive group. Unilateral lymphadenopathy found in 4 cases and 3 were on right side and 1 on left side and 2 were bilateral. Miliary shadow was found in 6 cases of which 2 patients $(16.67 \%)$ were suffering from HIV infection. Dholakia also reported mediastinal lymphadenopathy and miliary shadow more in HIV reactive patients. ${ }^{12}$ Kiyan E et al opine about cavity is slightly less in immunocompromised patients than non-immunocompromised and also hilar and mediastinal lymphadenopathy was also more frequent in immunocompromised patient. ${ }^{15}$ However Manikkam s et al did not find any statistical difference in x-ray chest patterns in HIV reactive or HIV non-reactive group. ${ }^{11}$

\section{CONCLUSION}

This study reveals that multiple cavitary lesions, consolidations and fibrosis are predominant findings in x-ray chest in HIV non-reactive group and hilar lymphadenopathy is predominantly found in HIV reactive group where cavities and consolidations were found to be comparatively less. Though cavitation in adult may raise the possibility of tuberculosis, it does not exclude MDR tuberculosis especially if it is multiple.

\section{ACKNOWLEDGEMENT}

We want to thank Dr. Abhijit Mukherjee, Assistant Professor, Community Medicine, Darjeeling, NBMC for his help in statistical analysis and Mr Santosh Shaw for his support while preparing the manuscript.

\section{REFERENCES}

[1] Global TB report. WHO 2017.

[2] UNAIDS. Report on the global AIDS epidemic: executive summary. UNAIDS/6-20E, 2006.

[3] Summary of findings of the $3^{\text {rd }}$ National health survey. India NFHS-3. International Institute of Population Science And Macro Inter Inc., 2005-6 (First assessed on Dec 2004).

[4] Chiang CY, Centis R, Migliori GB. Drug resistant tuberculosis: past, present, future. Respirology 2010;15(3):413-32.

[5] World Health Organizations. Global tuberculosis report, 2014. http://w.w.w who.int/tb/ publications/global_report/en/

[6] Orenstein EW, Basu S, Shah NS, et al. Treatment outcomes among patients with multi drug resistant tuberculosis: systemic review and meta-analysis. Lancet Infect Dis 2009;9(3):153-61.

[7] Icksan AG, Napitupulu MRS, Nawas MA, et al. Chest xray findings comparison between Multi drug resistant tuberculosis and drug sensitive tuberculosis. Journal of Natural Sciences, Biology and Medicines 2018;9(1):42-6.

[8] Deesuwan P, AutraviSittikul 0, Girapongsa L. Chest radiographic findings of multidrug resistant pulmonary tuberculosis in comparison to drug sensitive pulmonary tuberculosis in non-HIV patient. Region 4-5 Med J 2015;34:66-78.

[9] Cha J, Lee HY, Lee KS, et al. Radiological findings of extensively drug resistant tuberculosis in NON-AIDS adults: comparisons with findings of multidrugresistant and drug sensitive tuberculosis. Korean Journal of Radiology 2009;10(3):207-16.

[10] Chuchottaworn C, Thanachartwet V, Sanggsayunh P, et al. Risk factors for multidrug-resistant tuberculosis among patients with pulmonary tuberculosis at the central chest institute of Thailand. PLoS One 2015;10(10):e0139986.

[11] Manikkam S, Archary M, Bohat R. Chest x-ray patterns of pulmonary multi drug resistant tuberculosis in children in a high HIV prevalence setting. SA Journal of Radiology 2016;20(1) http://dx.doi.org/10.4102/sajr.v20i1.829

[12] Dholakia YN, D’Souza DT, Tolani MP, et al. Chest x-rays and associated clinical parameters in pulmonary tuberculosis cases for the National tuberculosis programme, Mumbai. Infect Dis Resp 2014;4:e10. 10.4081/idr.2012.34-38. 
[13] Long R. Drug resistant tuberculosis. CMAJ 2000;163(4):425-8.

[14] Pitchenik AC, Rubinson HA. The radiographic appearance of tuberculosis in patients with the Acquired Immunodeficiency Syndrome (AIDS) and pre AIDS. Am Rev Respir Dis 1985;131(3):393-6.
[15] Kiyan E, Eilicaslan Z, Gurgan M, et al. Clinical and radiographic features of pulmonary tuberculosis in non-AIDS immunocompromised patients. Int J Tuberc Lung Dis 2003;7(8):764-70.

[16] Nunes EA, De Capitani EM, Coelho E, et al. Patterns of anti- tuberculosis drug resistance among HIV-infected patients in Maputo, Mozambique, 2002-2003. Int J Tuberc Lung Dis 2005;9(5):494-500. 